\title{
Brane solutions sourced by a scalar with vanishing potential and classification of scalar branes
}

\author{
Mariano Cadoni, ${ }^{a, b}$ Edgardo Franzin $^{a, b, c}$ and Matteo Serra ${ }^{d}$ \\ ${ }^{a}$ Dipartimento di Fisica, Università di Cagliari, \\ Cittadella Universitaria, 09042 Monserrato, Italy \\ ${ }^{b}$ INFN, Sezione di Cagliari, \\ Cagliari, Italy \\ ${ }^{c}$ CENTRA, Departamento de Física, Instituto Superior Técnico, Universidade de Lisboa, \\ Avenida Rovisco Pais 1, 1049 Lisboa, Portugal \\ ${ }^{d}$ Dipartimento di Matematica, Sapienza Università di Roma, \\ Piazzale Aldo Moro 2, 00185 Roma, Italy \\ E-mail: mariano.cadoni@ca.infn.it, edgardo.franzin@ca.infn.it, \\ serra@mat.uniroma1.it
}

ABSTRACT: We derive exact brane solutions of minimally coupled Einstein-Maxwell-scalar gravity in $d+2$ dimensions with a vanishing scalar potential and we show that these solutions are conformal to the Lifshitz spacetime whose dual QFT is characterized by hyperscaling violation. These solutions, together with the AdS brane and the domain wall sourced by an exponential potential, give the complete list of scalar branes sourced by a generic potential having simple (scale-covariant) scaling symmetries not involving Galilean boosts. This allows us to give a classification of both simple and interpolating brane solution of minimally coupled Einstein-Maxwell-scalar gravity having no Schrödinger isometries, which may be very useful for holographic applications.

KEYwords: Black Holes, Holography and condensed matter physics (AdS/CMT)

ARXIV EPRINT: 1511.03986 


\section{Contents}

1 Introduction $\quad 1$

2 Einstein-Maxwell-scalar gravity 3

3 Scaling symmetries and hyperscaling violation 5

4 Brane solutions sourced by a scalar field with $V=0 \quad 6$

4.1 Uncharged brane solutions 6

4.1.1 Energy of the brane $\quad 7$

4.1.2 Scaling symmetries and hyperscaling violation 8

4.2 Electrically charged brane solutions in four dimensions 8

$\begin{array}{lll}\text { 4.2.1 Energy of the brane } & 10\end{array}$

$\begin{array}{lll}\text { 4.2.2 Scaling symmetries and hyperscaling violation } & 10\end{array}$

5 Classifications of brane solutions sourced by scalar fields $\quad 11$

$\begin{array}{lll}5.1 & \text { Elementary solutions } & 11\end{array}$

$\begin{array}{lll}5.2 & \text { Interpolating solutions } & 12\end{array}$

6 Domain-Wall/Conformal-Lifshitz interpolating solutions 14

7 Conformal-Lifshitz/Conformal-Lifshitz interpolating solutions $\quad 15$

$\begin{array}{lll}8 & \text { Conclusions } & 16\end{array}$

$\begin{array}{ll}\text { A Spherically symmetric solutions in four dimensions } & 17\end{array}$

\section{Introduction}

In its most general setting, Einstein-Maxwell-scalar gravity (EMSG) is characterized by three coupling functions: the self-interaction scalar potential $V(\phi)$, the gauge coupling function $Z(\phi)$, and the coupling function $S(\phi)$ responsible for the mass of the Maxwell field. In recent times, a lot of effort has been devoted to the derivation and investigation of brane solutions in the context of EMSG in $d+2$ dimensions with various forms of the coupling functions $[1-12]$.

The main reason behind this interest is the holographic application of this kind of solutions. Using the rules of the AdS/CFT correspondence [13-15], one can investigate the QFT dual to the brane and in particular derive its transport properties. This strategy has allowed to uncover a very rich phenomenology ranging from applications to condensed matter systems (holographic superconductors [16-19], phase transitions [4, 20], quantum 
criticality [11, 21, 22], hyperscaling violation in critical systems $[9,10,23-38]$, hydrodynamic regime of strongly coupled QFTs [39, 40], entanglement entropy [41-44]) to cosmology [45-49]. However, the essential qualitative features of the dual QFT are not pertinent to the presence of non-trivial coupling functions $Z$ and $S$ but are basically determined by the presence of a non-constant scalar field. In the dual QFT the non-trivial profile of the scalar field plays the crucial role of an order parameter triggering symmetry breaking and/or phase transitions. For this reason, in this paper we will restrict our considerations to minimally coupled Einstein-Maxwell-scalar gravity (MCEMSG), which is characterized by a single coupling function, the potential $V(\phi)$, whereas the other two are trivial, i.e. $Z=1$ and $S=0$.

The branes of MCEMSG have been investigated in several papers and both analytical and numerical solutions have been derived [1-12]. There are three different types of solutions. Black branes (BB) are solutions with a singularity shielded by an event horizon. In the dual QFT these solutions correspond to a field theory at finite temperature $T$ and are very important in the description of phase transitions [4, 9, 16-20]. Scale-covariant branes (SCB) are a sort of elementary solutions transforming covariantly under scale transformations. They represent a generalization of the usual Minkowski or AdS vacua to spacetimes with non-standard asymptotics $[6,23,50]$. They are sourced by a scalar field with $\log r$ behavior, have no horizon and in the dual QFT typically correspond to a $T=0$ ground state exhibiting hyperscaling violation $[9,10,23-31,34,35]$. The AdS brane, which is characterized by full conformal invariance and is sourced by a constant scalar, appears as limiting case of this class of solutions. Scale-covariant solutions in general have a curvature singularity at $r=0$ and need therefore an infrared (IR) regularization [27]. Interpolating branes are solutions which interpolate between two elementary branes at $r \sim 0$ (the IR of the dual QFT) and $r=\infty$ (the ultraviolet (UV) of the dual QFT). They are $T=0$ solutions describing the flow of the dual QFT from a IR to an UV regime, in which the solution behaves as an elementary, scale-covariant, solution.

The presence of a given type of brane solution in the spectrum of MCEMSG theories depends on the specific form of the potential $V(\phi)$. Elementary, scale-covariant branes require an exponential potential (a constant $V$ is required for the AdS brane), whereas black or interpolating branes typically require a more complicate potential with different behaviour in the $r=0$ and $r=\infty$ region. Notice that the known no-hair theorems [51-53] only apply to asymptotically flat or AdS solutions. Thus, solutions with non-standard asymptotics do not necessarily satisfy no-hair theorems. Although several solutions of MCEMSG theories are known, presently it is not clear if the scale-covariant geometries found until now exhaust all the possible solutions of this kind of theory. Clearly, this lack of knowledge prevents a complete classification of the possible interpolating geometries.

The purpose of this paper is twofold. First, we derive the exact solutions of MCEMSG in the case of a vanishing potential, which are the brane counterpart of the Janis-NewmannWinicour-Wyman (JNWW) solutions [54-57]. We show that these solutions belong to the class of scale-covariant solutions generating hyperscaling violation in the dual QFT. Second, we demonstrate that these solutions complete the list of the possible scale-covariant solutions of the theory having isometries not involving Galilean boosts. This will allow us 
to give an exhaustive classification of the interpolating brane solutions of MCEMSG with no Schrödinger isometries [58-63].

The structure of this paper is the following. In section 2 we briefly review MCEMSG and the parametrization of the field equations introduced in ref. [6]. In section 3 we classify the branes according to their hyperscaling violation parameter and dynamic scaling exponent. We then derive and discuss the brane solutions of the theory with a vanishing potential in section 4 . In section 5 we show that this solution completes the list of elementary, scale-covariant branes of the theory and we give a complete classification of both elementary and interpolating solutions. In section 6 and section 7 we construct two explicit examples of interpolating solutions. Finally, we conclude in section 8. In appendix A we present a result, which is somehow beyond the scope of this paper, but can be obtained using our parametrization of the field equations, namely the charged, spherically symmetric solutions of MCEMSG. These solutions are the charged generalization of the JNWW solutions.

\section{Einstein-Maxwell-scalar gravity}

We consider MCEMSG in $d+2$ spacetime dimensions (with $d \geqslant 2$ ):

$$
A=\int d^{d+2} x \sqrt{-g}\left(\mathcal{R}-2(\partial \phi)^{2}-F^{2}-V(\phi)\right),
$$

where $\mathcal{R}$ is the scalar curvature of the spacetime. The ensuing field equations are

$$
\begin{aligned}
\nabla_{\mu} F^{\mu \nu}= & 0 \\
\nabla^{2} \phi= & \frac{1}{4} \frac{d V(\phi)}{d \phi}, \\
\mathcal{R}_{\mu \nu}-\frac{1}{2} g_{\mu \nu} \mathcal{R}= & 2\left(F_{\mu \rho} F_{\nu}^{\rho}-\frac{1}{4} g_{\mu \nu} F^{\rho \sigma} F_{\rho \sigma}\right) \\
& +2\left(\partial_{\mu} \phi \partial_{\nu} \phi-\frac{1}{2} g_{\mu \nu} \partial^{\rho} \phi \partial_{\rho} \phi\right)-\frac{1}{2} g_{\mu \nu} V(\phi) .
\end{aligned}
$$

We are interested in static, radially symmetric solutions of the field equations, for this we parametrize the spacetime metric in a Schwarzschild gauge:

$$
d s^{2}=-U(r) d t^{2}+U^{-1}(r) d r^{2}+R^{2}(r) d \Omega_{(\varepsilon, d)}^{2},
$$

where $\varepsilon=0,1,-1$ denotes, respectively, the $d$-dimensional planar, spherical, or hyperbolic transverse space with metric $d \Omega_{(\varepsilon, d)}^{2}$. Moreover, we will consider only purely electric solutions. ${ }^{1}$ Let $Q$ be the electric charge. Then, the electric field satisfying (2.2) reads

$$
F_{t r}=\frac{Q}{R^{d}}
$$

\footnotetext{
${ }^{1}$ For $d=2$ magnetic solutions can be easily generated from the electric ones using the electro-magnetic duality. In this case we have $F_{i j}=\frac{Q}{R^{2}} \epsilon_{i j}$, where $i, j$ label the transverse two-dimensional space.
} 
Using eqs. (2.3) and (2.4), the field equations take the form

$$
\begin{aligned}
\frac{R^{\prime \prime}}{R} & =-\frac{2}{d}\left(\phi^{\prime}\right)^{2}, \\
\left(U R^{d} \phi^{\prime}\right)^{\prime} & =\frac{1}{4} R^{d} \frac{d V}{d \phi}, \\
\left(U R^{d}\right)^{\prime \prime} & =\varepsilon d(d-1) R^{d-2}+2 \frac{d-2}{d} \frac{Q^{2}}{R^{d}}-\frac{d+2}{d} R^{d} V, \\
\left(U R^{d-1} R^{\prime}\right)^{\prime} & =\varepsilon(d-1) R^{d-2}-\frac{2}{d} \frac{Q^{2}}{R^{d}}-\frac{1}{d} R^{d} V .
\end{aligned}
$$

In general, the solutions of these field equations depend on the class of potentials $V(\phi)$ considered. Usually, one must impose precise boundary conditions on the $r=\infty$ asymptotic behavior of the solution, which translate into boundary conditions for the potential. For example, if we require an asymptotically flat spacetime and assume without loss of generality that $\phi(r \rightarrow \infty)=0$, it follows that $V(0)=0$, while for asymptotically AdS spacetimes we have $V(0)=-\Lambda^{2}$. Typically one also requires the existence of the Schwarzschild black hole (black brane) solution sourced by a constant scalar field $\phi=0$, implying $V^{\prime}(0)=0$, while the existence of black hole solutions sourced by a non-trivial scalar field in general is strongly constrained by well-known no-hair theorems.

Nevertheless, finding exact solutions of the field equations (2.5) is a very difficult task even when the explicit form of the potential $V$ is given. An efficient solving method has been proposed in ref. [6]. Such method is particularly useful in the holographic context and it has been successfully used in several cases to generate exact, asymptotically flat or AdS, solutions of Einstein-(Maxwell)-scalar gravity, where the potential is not an input but an output of the theory $[6,56,57]$.

Following ref. [6], introducing the variables $R=e^{\int Y}$ and $u=U R^{d}$, the field equations (2.5) become

$$
\begin{aligned}
Y^{\prime}+Y^{2} & =-\frac{2}{d}\left(\phi^{\prime}\right)^{2}, \\
\left(u \phi^{\prime}\right)^{\prime} & =\frac{1}{4} e^{d \int Y} \frac{d V}{d \phi}, \\
u^{\prime \prime}-(d+2)(u Y)^{\prime} & =-2 \varepsilon(d-1) e^{(d-2) \int Y}+4 Q^{2} e^{-d \int Y}, \\
u^{\prime \prime} & =\varepsilon d(d-1) e^{(d-2) \int Y}+2 \frac{d-2}{d} Q^{2} e^{-d \int Y}-\frac{d+2}{d} e^{d \int Y} V .
\end{aligned}
$$

Eqs. (2.6c) and (2.6d) are second-order linear differential equations in $u$, whereas (2.6a) is a first-order nonlinear equation for $Y$, known as the Riccati equation. In general, starting from a given profile $\phi(r)$ for the scalar field, one can look for solutions of the Riccati equation. Once the solution for $Y$ has been found, one can integrate eq. (2.6c), which is linear in $u$, to obtain

$$
u=R^{d+2}\left[\int\left(4 Q^{2} \int \frac{1}{R^{d}}-2 \varepsilon(d-1) \int R^{d-2}-C_{1}\right) \frac{1}{R^{d+2}}+C_{2}\right],
$$


where $C_{1,2}$ are integration constants. The last step is the determination of the potential using eq. (2.6d):

$$
V=\frac{d^{2}(d-1)}{d+2} \frac{\varepsilon}{R^{2}}+2 \frac{d-2}{d+2} \frac{Q^{2}}{R^{2 d}}-\frac{d}{d+2} \frac{u^{\prime \prime}}{R^{d}}
$$

\section{Scaling symmetries and hyperscaling violation}

The $d+2$ dimensional metric for a brane $(\varepsilon=0)$, whose dual QFTs are characterized by hyperscaling violation is usually written as [23]

$$
d s^{2}=r^{-2(d-\theta) / d}\left(-r^{-2(z-1)} d t^{2}+d r^{2}+d x_{i} d x^{i}\right),
$$

where $d x_{i} d x^{i}=d \Omega_{(0, d)}^{2}, \theta$ is the hyperscaling violation parameter and $z$ is the dynamic scaling exponent characterizing the anisotropic scaling of the time and space coordinates, which breaks Lorentz invariance in the dual QFT. The scaling symmetries of the metric (3.1) are

$$
t \rightarrow \lambda^{z} t, \quad r \rightarrow \lambda r, \quad x_{i} \rightarrow \lambda x_{i}, \quad d s \rightarrow \lambda^{\theta / d} d s
$$

It follows that a nonzero value of $\theta$ makes the metric (3.1) not scale-invariant, but only scale-covariant, in the sense that the metric transforms with a definite weight under a scale transformation. Notice that in this paper we consider simple scaling symmetries (3.2), which do not involve Galilean boosts. Holography for non-relativistic QFTs have been also investigated in the case in which the geometry allows for scaling isometries involving boosts (Schrödinger isometries). In this case the theory has not a preferred rest frame as the simple scaling geometries (3.1) and we have an holographic description which has been termed Schrödinger holography [58-63]. A complete and exhaustive classification of all non-relativistic holographic models, in particular of the important case $z=2$, would require a separate classification involving also Schrödinger symmetries. Such a complete classification is, however, beyond the scope of this paper.

The scaling transformation determines the following scaling behavior for the free energy of the dual QFT, given in terms of $\theta$ and $z$ :

$$
F \sim T^{\frac{d+z-\theta}{z}}
$$

It is useful to distinguish between the two effects of hyperscaling violation $(\theta \neq 0)$ and anisotropic scaling $(z \neq 1)$ introducing four different subclasses:

(1) $\theta=0, z=1$ describes AdS branes. The metric (3.1) gives the AdS spacetime in $d+2$ dimensions, the scaling (3.2) is isotropic and the dual QFT is a CFT.

(2) $\theta=0, z \neq 1$ describes Lifshitz branes. Because $\theta=0$, the metric is not only covariant but also invariant under the scale transformation (3.2). On the other hand, because $z \neq 1$ the scaling is not isotropic in the $t$ and $x_{i}$ coordinates and the dual $d+1$ dimensional QFT is not invariant under the $d+1$-dimensional Lorentz group. 
(3) $\theta \neq 0, z=1$ describes Domain Walls. The scaling (3.2) is isotropic but being $\theta \neq 0$ full scale invariance is broken and only scale covariance survives. The metric is conformal to AdS spacetime. The dual QFT is Lorentz invariant and one can still formulate a DW/QFT correspondence $[64,65]$. Notice that Minkowski spacetime in $d+2$ dimensions is a particular case of this class of solutions, it is obtained for $\theta=d$.

(4) $\theta \neq 0, z \neq 1$ describes Conformal-Lifshitz branes. Now the scaling (3.2) is anisotropic and hyperscaling is violated. In this case the metric (3.1) is conformal to the Lifshitz spacetime [6].

Hyperscaling violation can be realized both for $\theta>0$ and $\theta<0$. The two cases are qualitative different. As one can infer from the scaling of the free energy (3.3), the effect of a positive hyperscaling violation parameter $\theta$ is a "lowering" of the effective dimensionality of the dual system from $d$ to $d-\theta$, whereas for $\theta<0$ this dimensionality increases to $d+|\theta|$. For usual condensed matter critical systems $\theta$ is always positive. However, in the context of holographically generated hyperscaling violation, negative values of $\theta$ have been found in a number of cases, see e.g. ref. $[9,10]$ and references therein.

\section{Brane solutions sourced by a scalar field with $V=0$}

For an identically vanishing potential, $V(\phi)=0$, it is possible to find exact solutions of the field equations (2.2). The electrically neutral, spherically symmetric JNWW solutions [54, 55] have been rederived in ref. [56] using the method described in section 2.

In this paper we will focus our attention on solutions in $d+2$ spacetime dimensions for which $\varepsilon=0$ (branes), i.e. the transverse $d$-dimensional sections have planar topology. In this case the field equations eq. (2.6) reduce to

$$
\begin{aligned}
Y^{\prime}+Y^{2} & =-\frac{2}{d}\left(\phi^{\prime}\right)^{2}, \\
\left(u \phi^{\prime}\right)^{\prime} & =0, \\
u^{\prime \prime}-(d+2)(u Y)^{\prime} & =4 Q^{2} e^{-d \int Y}, \\
u^{\prime \prime} & =2 \frac{d-2}{d} Q^{2} e^{-d \int Y .}
\end{aligned}
$$

Let us discuss separately the uncharged $(Q=0)$ and charged case.

\subsection{Uncharged brane solutions}

We consider first the electrically neutral case $Q=0$. One can easily realize that flat branes with $R=r$ and $U=1$ (corresponding to $u=r^{d}, Y=1 / r$ ), are not solution of the field equations (4.1). However, the system (4.1) can be integrated exactly: we solve the trivial equation (4.1d), giving $u$ as a linear function of $r$; then we solve eq. (4.1c) for $Y$; and finally we determine $\phi$ using eq. (4.1b). The Riccati equation then gives just a constraint for the integration constants. The most general form of the solution is:

$$
U=\left(\frac{r}{r_{0}}\right)^{1-d w}, \quad R^{2}=\left(\frac{r}{r_{0}}\right)^{2 w}, \quad \phi=-\gamma \log \left(\frac{r}{r_{0}}\right)+\phi_{0}, \quad w-w^{2}=\frac{2}{d} \gamma^{2},
$$


where $r_{0}, \gamma, w$ and $\phi_{0}$ are integration constants. The constraint implies the condition $0 \leqslant w \leqslant 1$. The solution (4.2) is invariant under the transformation $w \rightarrow 1-w$, which maps solutions with $w \in[0,1 / 2]$ into solutions with $w \in[1 / 2,1]$. Neglecting the constant $\phi_{0}$, a trivial translation mode of the scalar, these solutions give a two-parameter $\left(r_{0}, w\right)$ family of brane solutions. In particular, $r_{0}$ represents a length scale, while $w$ is a dimensionless parameter. The solution (4.2) has interesting scaling symmetries, which we discuss in detail in subsection 4.1.2. Moreover, it can be considered as the brane counterpart of the JNWW spherical solutions.

For $w \neq 0,1$ the solutions have a naked singularity at $r=0$, in fact the scalar curvature is

$$
\mathcal{R}=\frac{2 \gamma^{2}}{r_{0}^{2}}\left(\frac{r}{r_{0}}\right)^{-1-d w} .
$$

Well-known no-hair theorems [51-53] forbid asymptotically flat BB solutions when $V=0$. In principle these theorems do not apply to solutions with non-standard asymptotics such as (4.2). Nevertheless, one can easily check that eq. (4.1) do not allow for solutions with event horizons. Thus, the brane (4.2) is a $T=0$ solution, which does not allow for finite temperature excitations.

In the two limiting cases $w=0,1$ the scalar is constant. For $w=0$ the brane solution becomes

$$
d s^{2}=-\frac{r}{r_{0}} d t^{2}+\frac{r_{0}}{r} d r^{2}+d x_{i} d x^{i}
$$

which is just $(d+2)$-dimensional Minkowski space in a particular coordinate system; in fact, the $(r, t)$ sections of the metric can be brought in the Rindler form by a simple redefinition of the radial coordinate $r$. Whereas, for $w=1$ the brane solution is

$$
d s^{2}=-\left(\frac{r}{r_{0}}\right)^{1-d} d t^{2}+\left(\frac{r}{r_{0}}\right)^{d-1} d r^{2}+\left(\frac{r}{r_{0}}\right)^{2} d x_{i} d x^{i},
$$

a Ricci-flat manifold, which can be considered as the brane counterpart of the Schwarzschild black hole.

\subsubsection{Energy of the brane}

Let us now calculate the total energy $M$ of the solution, using the Euclidean action formalism of ref. [66]. The variation of the boundary terms of the action consists of both a gravitational and a scalar contribution:

$$
\delta M=8 \pi\left[-\frac{d}{2} R^{d-1} R^{\prime} \delta U+U^{\prime} R^{d / 2} \delta\left(R^{d / 2}\right)-d U R^{d / 2} \delta\left(R^{\frac{d-2}{2}} R^{\prime}\right)\right]^{\infty}-16 \pi\left[R^{d} U \phi^{\prime} \delta \phi\right]^{\infty} .
$$

Evaluating this equation for the solution (4.2) one finds

$$
M=\frac{4 \pi}{r_{0}} d w[(2-d) w-2] .
$$


Taking into account the constraints on $w$ and $d$ (namely $0 \leqslant w \leqslant 1$ and $d \geqslant 2$ ), the energy vanishes only when $w=0$, i.e. for Minkowski space, while for $0<w \leqslant 1$ the sign of the energy is ruled by the sign of $r_{0}$ (negative for $r_{0}>0$ and positive for $r_{0}<0$ ). Taking $r>0$, solution (4.2) exists only for $r_{0}>0$, thus the brane has always negative mass. This behaviour is quite different from the JNWW solutions, where the sign of the energy depends both on the value of the dimensionless parameter of the solutions and on $r_{0}[56,57]$.

\subsubsection{Scaling symmetries and hyperscaling violation}

The brane metric (4.2) has remarkable scaling symmetries and can be put in the form (3.1) by the transformation of coordinates

$$
\frac{r}{r_{0}} \rightarrow\left(\frac{r}{\tilde{r}_{0}}\right)^{\frac{2}{1+w(d-2)}}, \quad \tilde{r}_{0}=\frac{2 r_{0}}{1+w(d-2)} .
$$

The hyperscaling violation parameter and the dynamical exponent are given by

$$
z=\frac{2 d w}{1+w(d-2)}, \quad \theta=\frac{d(1+d w)}{1+w(d-2)} .
$$

We observe that $z$ and $\theta$ are not independent but satisfy the relation

$$
\theta=z+d
$$

From eq. (4.9) we see that the brane solution (4.2) never belongs to the subclasses (1) or (2) of section 3, i.e. it can neither describe an AdS spacetime nor a Lifshitz spacetime. It can be either a DW for $w=1 /(d+2)$ or a CL brane for $w \neq 1 /(d+2)$. The relation $(4.10)$ implies that the free energy of the dual QFT is constant. This is what we expect because, owing to the absence of solutions with event horizons, our brane solution does not allow $\mathrm{BB}$ excitations at finite temperature.

The null energy conditions for the bulk stress-energy tensor require [23]

$$
(d-\theta)(d(z-1)-\theta) \geqslant 0, \quad(z-1)(d+z-\theta) \geqslant 0 .
$$

Taking into account that the constraint $0 \leqslant w \leqslant 1$ implies the condition $0 \leqslant z \leqslant 2 d /(d-1)$, it is straightforward to check that the first inequality is always satisfied. The second one is saturated in our case, as expected because the source is a massless field.

\subsection{Electrically charged brane solutions in four dimensions}

Let us now consider the case of non-vanishing electric charge. Taking for simplicity $d=2$, eq. (4.1) become

$$
Y^{\prime}+Y^{2}=-\left(\phi^{\prime}\right)^{2}, \quad\left(u \phi^{\prime}\right)^{\prime}=0, \quad u^{\prime \prime}-4(u Y)^{\prime}=4 Q^{2} e^{-2 \int Y}, \quad u^{\prime \prime}=0 .
$$


Again, we first determine $u$ and $\phi$, then we solve the Riccati equation, and finally we use the third equation to express the charge $Q$ in terms of the integration constants:

$$
\begin{aligned}
U & =\left(\frac{r}{r_{0}}\right)^{1-2 w}\left[1-c_{1}\left(\frac{r}{r_{0}}\right)^{1-2 w}\right]^{-2}, \\
R^{2} & =\left(\frac{r}{r_{0}}\right)^{2 w}\left[1-c_{1}\left(\frac{r}{r_{0}}\right)^{1-2 w}\right]^{2}, \\
\phi & =-\gamma \log \left(\frac{r}{r_{0}}\right)+\phi_{0}
\end{aligned}
$$

where $w-w^{2}=\gamma^{2}$ and $c_{1}=r_{0}^{2} Q^{2} /(1-2 w)^{2}$. These constraints imply $0 \leqslant w \leqslant 1$, with $w \neq 1 / 2$.

Solutions (4.13) represent a three-parameter $\left(r_{0}, w, Q\right)$ family of brane solutions, with a naked singularity at $r=r_{0} c_{1}^{(1 / 2 w-1)}$ for all $w$ in the range $0 \leqslant w \leqslant 1$. In fact the scalar curvature is

$$
\mathcal{R}=\frac{2 \gamma^{2}}{r_{0}^{2}}\left(\frac{r}{r_{0}}\right)^{-1-2 w}\left[1-c_{1}\left(\frac{r}{r_{0}}\right)^{1-2 w}\right]^{-2} .
$$

For $w=0,1$ the solutions reduce to curved branes with vanishing scalar curvature $\mathcal{R}=0$, sourced by a constant scalar field. In particular one has:

$$
\begin{array}{lll}
U=\frac{r}{r_{0}}\left(1-\frac{c_{1} r}{r_{0}}\right)^{-2}, & R=1-\frac{c_{1} r}{r_{0}} & \text { for } w=0, \\
U=\frac{r_{0}}{r}\left(1-\frac{c_{1} r_{0}}{r}\right)^{-2}, & R=\frac{r}{r_{0}}-c_{1} & \text { for } w=1 .
\end{array}
$$

Both solutions represent a sort of Reissner-Nordström (RN) branes. In fact, the metric part of the solutions can be written using a translation and a rescaling of the radial coordinate $r$ in the form $U=\frac{\alpha}{r}+\frac{Q^{2}}{r^{2}}, R=r$, where $\alpha$ is a constant.

The asymptotic behavior of solution (4.13) in general is ruled by the value of $w$. When $0<w<1 / 2$, we have for $r \rightarrow \infty$ (corresponding to $\phi \rightarrow-\infty$ ) at leading order:

$$
U=c_{1}^{-2}\left(\frac{r}{r_{0}}\right)^{-1+2 w}, \quad R^{2}=c_{1}^{2}\left(\frac{r}{r_{0}}\right)^{2-2 w}, \quad \phi=-\gamma \log \frac{r}{r_{0}},
$$

while when $1 / 2<w<1$ one finds:

$$
U=\left(\frac{r}{r_{0}}\right)^{1-2 w}, \quad R^{2}=\left(\frac{r}{r_{0}}\right)^{2 w}, \quad \phi=-\gamma \log \frac{r}{r_{0}} .
$$

Notice that in this last case the asymptotic behavior coincides with the uncharged solution (4.2) with $d=2$, discussed in the previous subsection. It is also easy to check that the two asymptotic forms (4.17) and (4.18) are mapped one into the other by the transformation $w \rightarrow 1-w$, that leaves invariant the constraint $w-w^{2}=\gamma^{2}$. 


\subsubsection{Energy of the brane}

Also in this case, we can compute the total energy of the solution using the Euclidean action formalism [67]. The variation of the boundary terms of the action are:

$$
\delta M=\delta M_{G}+\delta M_{\phi}-\left.16 \pi Q \delta \Phi\right|^{\infty},
$$

where $\delta M_{G}$ and $\delta M_{\phi}$ are the gravitational and scalar contributions, corresponding to eq. (4.6) with $d=2$, while the last term is the contribution due to the charge and $\Phi$ is the electric potential.

Evaluating the energy for our solution (4.13), we find that the electromagnetic contribution always vanishes at $r=\infty$, while the full result is ruled by the value of $w$ again. When $0 \leqslant w \leqslant 1 / 2$ we obtain

$$
M=\frac{16 \pi}{r_{0}}\left[-w+(2 w-1) \log \left(Q / Q_{0}\right)\right],
$$

where $Q_{0}$ is an integration constant. In this case the sign of the energy depends on the mutual values of $w$ and $Q$. When $1 / 2 \leqslant w \leqslant 1$ (where the asymptotic behavior of the charged brane (4.13) coincides with the uncharged brane (4.2) for $d=2$ ), one simply finds

$$
M=-\frac{16 \pi}{r_{0}} .
$$

As expected, it coincides with the energy (4.7) of the uncharged solution with $d=2$.

\subsubsection{Scaling symmetries and hyperscaling violation}

Let us now study the scaling symmetries of the solution (4.13) in the UV regime, i.e. for $r \rightarrow \infty$. We consider first the asymptotic form (4.17), describing the UV regime of the solution when $0 \leqslant w \leqslant 1 / 2$. Let $\tilde{r}_{0}=2 r_{0} c_{1}$, then (4.17) can be put in the form (3.1) by the transformation of coordinates

$$
\frac{r}{r_{0}} \rightarrow\left(\frac{r}{\tilde{r}_{0}}\right)^{2}
$$

After this transformation, it is simple to extract the hyperscaling violation parameter and the dynamical exponent:

$$
z=4-4 w, \quad \theta=6-4 w .
$$

From eq. (4.23) one can see that $\theta=0$ for $w=3 / 2$, which is outside the range of $w$. Hence, our brane solution (4.13) cannot describe neither an AdS nor a Lifshitz brane, i.e. it never belongs to the subclasses (1) or (2) of section 3. The brane is either a DW for $w=3 / 4$ or a CL brane for $w \neq 3 / 4$. Notice also that the relation $\theta=z+2$ (already observed in the uncharged case in its $d$-dimensional form), is verified again, as well as the constant free energy of the dual QFT.

The null energy conditions for the bulk stress-energy tensor

$$
(2-\theta)[2(z-1)-\theta] \geqslant 0, \quad(z-1)(2+z-\theta) \geqslant 0
$$

are satisfied. In particular, the second one is saturated.

Exploiting the symmetry of the asymptotic solutions (4.17) and (4.18) under $w \rightarrow 1-w$ described in section 4.2 , we can easily derive the critical exponents $\theta$ and $z$ related to the scaling (4.18), which, as expected, are those of (4.9) with $d=2$. 


\section{Classifications of brane solutions sourced by scalar fields}

In this section we present a detailed physical classification of brane solutions sourced by a scalar field with a self-interaction potential $V$, i.e. a classification of the brane solutions of the MCEMSG theory (2.1). Obviously, the form of the brane solution will strongly depend on the form of the potential $V$ and a complete classification is without reach. On the other hand, having in mind holographic applications, we are not interested in generic solutions but on branes that respect some scaling symmetries, at least asymptotically $(r \rightarrow \infty)$ and in the $r=0$ region. We can therefore built up a brane classification based on the scaling symmetries discussed in section 3 .

We will preliminary show that the scale-covariant solution (4.2) together with the solutions already known in the literature give all the possible brane solutions of MCEMSG of the form (3.1). Using a reparametrisation of the radial coordinate $r$ we can easily write the metric (3.1) in the form (2.3) with $U=B\left(r / r_{0}\right)^{\alpha}, R=D\left(r / r_{0}\right)^{\beta}$, from which it follows $Y=\beta / r$. Inserting this expression of $Y$ in the field equations (2.6) with $Q=0$ one finds only three classes of solutions:

(I) $\beta=1, \phi=$ const, $u \propto r^{d+2}, V=-\Lambda^{2}$, which corresponds to the $\mathrm{AdS}_{d+2}$ brane;

(II) $\phi \propto \log \left(r / r_{0}\right), u \propto r, V=0$, which corresponds to the solution (4.2);

(III) $\phi \propto \log \left(r / r_{0}\right), u \propto r^{\eta}, V \propto e^{\mu \phi}, \eta \neq\{1, d+2\}$, which corresponds to DW solutions sourced by an exponential potential [6].

In our classification, we will distinguish between elementary solutions, i.e. solutions respecting some scaling symmetry, and interpolating solutions, i.e. solutions that approach to elementary branes only in the $r=0$ IR region and in the $r=\infty$ UV region. We will discuss separately these two types of solutions.

\subsection{Elementary solutions}

Elementary solutions are defined as those solutions of the theory (2.1) which belong to one of the subclasses of section 3. In principle, we should therefore have in correspondence with the four scale symmetries of section 3 four kinds of elementary branes. However, as a consequence of the previous demonstration, the Lifshitz solution (2) cannot be obtained if the source is a minimally coupled scalar field. We are therefore left with three classes of solutions:

(A) AdS branes are $Q=0$ solutions of the model (2.1) when the potential $V(\phi)$ is a negative cosmological constant or has a local extremum $V^{\prime}\left(\phi_{0}\right)=0$, with $V\left(\phi_{0}\right)=$ $-\Lambda^{2}$. In this case we have a trivial (constant) scalar field $\phi=\phi_{0}$.

(B) Domain Walls are $Q=0$ solutions of the model (2.1) when the potential $V(\phi)$ is a pure exponential: $V(\phi) \propto e^{\mu \phi}$. DWs are sourced by a scalar behaving logarithmically: $\phi \propto \log r / r_{0}$ [6] 
(C) Conformal-Lifshitz branes are solutions of the model (2.1) in the case $V=0$ for $Q=0$. For $Q \neq 0$ they appear as solution of the theory for a purely exponential potential [6].

For $z$ and $\theta$ in eq. (3.1) finite, there are no other elementary brane solutions which can be sourced by a minimally coupled scalar field. However, in the $\theta=0, z \rightarrow \infty$ limit, it is known that the Lifshitz brane becomes the $\mathrm{AdS}_{2} \times R_{d}$ spacetime, see e.g. ref. [27]. If one wants to include this limiting case one should consider also a fourth kind of elementary brane solutions, namely the $A d S_{2} \times R_{d}$ branes. These spacetimes are $Q \neq 0$ charged solutions of the model (2.1) when the potential $V(\phi)$ is a negative cosmological constant or has a local extremum $V^{\prime}\left(\phi_{0}\right)=0$, with $V\left(\phi_{0}\right)=-\Lambda^{2}$. Similarly to case (A) these branes are sourced by a constant scalar field.

\subsection{Interpolating solutions}

Combining the three types of elementary brane solutions discussed above one can construct different kinds of interpolating solutions, i.e. solutions behaving only in the UV and IR regimes as an elementary brane. The interpolating solutions are very useful for holographic applications, in particular for AdS/CFT and the gravity/condensed matter correspondence of EMSG [1-12].

The recent literature dealing with this topic contains a multitude of such interpolating brane solutions derived in the context of the gravity theory (2.1) and its possible generalizations (covariant coupling between the U(1) gauge field $A_{\mu}$ and the scalar, coupling between the Maxwell tensor $F_{\mu \nu}$ and the scalar, Einstein-Yang-Mills-scalar gravity, etc.). Despite this variety of solutions and models, the simplest case described by the action (2.1) is extremely important for the crucial role played by the scalar field. In the dual QFT the scalar field gives an order parameter triggering symmetry breaking and/or phase transitions. Moreover, $\phi$ has a nice interpretation in terms of holographic renormalization group equations describing the flow between UV/IR fixed points, see e.g. ref. [68].

The classification of the possible interpolating solutions of the theory (2.1) is simple because it is parametrized by a single function, the potential $V(\phi)$. It follows that the interpolating solutions are essentially determined by the behaviour of the potential in the IR and UV region. This feature is not present in other, more complicated, models in which the presence of two or more coupling functions prevents a simple classification. In the following we will list all the known interpolating solutions and, in the case they are not been already discussed in the literature, we will discuss their possible existence.

AdS-AdS interpolating solutions In general, solutions of this kind are present when the potential has a local maximum and a local minimum connected with continuity. The gravitational soliton bridges two AdS spacetimes whereas the dual field theory flows from a fixed point in the UV to an other fixed point in the IR. The two CFTs are connected by the c-theorem, which gives well-defined predictions for the running of the central charge when running from the UV to the IR. Interpolating solutions of this kind are typically numerical solutions and have been already discussed in the literature, see e.g. ref. [69]. 
AdS-DW Interpolating solutions Typically, these solutions are present when $Q=0$ and the potential has an extremum at $\phi_{0}$ with $V\left(\phi_{0}\right)<0$ in the UV (IR), whereas it behaves exponentially in the IR (UV). The gravitational soliton interpolates between an AdS spacetime at $r=\infty(r=0)$ and a DW near $r=0(r=\infty)$. The dual QFT flows from a fixed point in the UV (IR) to an hyperscaling violating phase in the IR (UV). Exact solutions of this kind are known, both in the case of hyperscaling violation in the IR [6] and hyperscaling violation in the UV [8, 9]. Several numerical solutions are also known, see e.g. ref. [12].

AdS-CL Interpolating solutions Brane solutions of the theory (2.1) bridging an AdS spacetime in the UV (IR) with a CL solution in the IR (UV) have not been discussed in the literature. Conversely, they are quite common in non-minimally coupled theories and in the case of holographic superconductors. In the context of the minimally coupled theory they are expected to show up in two cases: first, $V(\phi)$ has an extremum in the UV (IR) whereas in the IR (UV) region the kinetic energy of the scalar dominates over its potential energy so that we can use $V \sim 0$; second, we have $Q \neq 0$ charged solutions, $V(\phi)$ has an extremum in the UV (IR) whereas in the IR (UV) region $V$ behaves exponentially. Obviously the existence of these solutions must be checked numerically.

DW-DW Interpolating solutions Solutions interpolating between two DW branes are not known in literature. However, we can easily find a form of the potential which is a good candidate for generating this kind of solution. One can start from a simple combination of exponentials: $V(\phi)=A e^{\alpha \phi}+B e^{-\beta \phi}$, that obviously behaves as a single exponential in the two regimes $\phi \rightarrow \infty$ and $\phi \rightarrow-\infty$. We know that a simple exponential form of the potential leads, in the case of uncharged branes, to a DW solution [6]. Thus, the corresponding brane solutions of the model, if existing would give a soliton interpolating between two DWs at $\phi= \pm \infty$.

DW-CL Interpolating solutions Having in mind the features of the elementary solutions discussed in section 5.1, one can expect this kind of solution to show up in the case of a potential which diverges exponentially in a region whereas approaches to zero in an other region. Solutions of this type, although already known in the literature [6], have not been recognized as DW-CL interpolating solutions. We will show in section 6 that for an appropriate choice of the parameters the solutions of ref. [6] describe a DW-CL interpolating solution.

CL-CL Interpolating solutions In order to generate these kind of branes one should consider a potential which vanishes in two distinct regions. Alternatively one can consider charged solutions and a potential behaving exponentially. Also a mixed charged configuration, with a potential vanishing in one region and behaving exponentially in an other region, is possible. Also solutions of this type are known in literature [6], but have not been recognized as CL-CL interpolating solutions. We will show in section 7 that for an appropriate choice of the parameters the solutions of ref. [6] describe a CL-CL interpolating solution. 
In the above classification of interpolating brane solutions we have not considered the limiting case in which one of the elementary solution is $\mathrm{AdS}_{2} \times R_{d}$. Brane solutions interpolating between an elementary solution (A), (B) or (C) in the UV and $\mathrm{AdS}_{2} \times R_{d}$ in the IR possibly exist whenever one considers charged branes and a potential $V$ behaving in the IR as a negative cosmological constant. The simplest, well-known, example of this kind is obtained considering $V=-\Lambda^{2}$ identically. The charged brane solutions are simply given by the AdS-RN BB. In the extremal limit, when the BPS bound is saturated, we get a solitonic solution which interpolates between $\mathrm{AdS}_{d+2}$ in the $r \rightarrow \infty$ region and $\mathrm{AdS}_{2} \times R_{d}$ in the near-horizon region. The $\mathrm{AdS}_{2} \times R_{d}$ geometry and related interpolating solutions are of interest also because they may act as IR regulators of the generic scale-covariant geometry (3.1) [27].

All the above interpolating solutions are considered as branes without event horizons, i.e. as zero temperature solutions. An important question, particularly in view of holographic applications, is if they can be considered as the extremal limit of BB solutions with non-trivial hair, i.e. solutions at finite temperature endowed with a non-trivial scalar field. There is no general answer to this question. Owing to no-hair theorems [51-53] the existence of hairy solutions is related to global features of the potential $V(\phi)$. Nevertheless, in most examples discussed in the literature the interpolating solutions appear as extremal limit of BB solutions.

\section{Domain-Wall/Conformal-Lifshitz interpolating solutions}

In this section we discuss exact solutions, which interpolate between a DW and a CL brane. In the previous section we have seen that this kind of solution requires uncharged branes and a potential which diverges exponentially in a region, whereas approaches to zero in an other region. We are therefore lead to consider $Q=0$ solutions and the following simple potential $(0 \leqslant w \leqslant 1)$

$$
V(\phi)=A d w(1-w(d+2)) e^{-\sqrt{\frac{8(1-w)}{d w}} \phi},
$$

which diverges exponentially for $\phi \rightarrow-\infty$, while $V \rightarrow 0$ for $\phi \rightarrow \infty$. The general solution for the theory $(2.1)$ with this potential is given by $[6]$

$$
U=A R^{2}-c\left(\frac{r}{r_{0}}\right)^{1-d w}, \quad R^{2}=\left(\frac{r}{r_{0}}\right)^{2 w}, \quad \phi=\sqrt{\frac{d}{2}\left(w-w^{2}\right)} \log \left(\frac{r}{r_{0}}\right),
$$

where $0 \leqslant w \leqslant 1$ and $c$ is an integration constant. Notice that when $A=0$ and $c<0$ the solution (6.2) becomes exactly the solution (4.2). This is consistent with the fact that for $A=0$, the potential is identically zero and the solution is sourced by the kinetic term of the scalar.

When $c>0$ and for $1 /(d+2)<w \leqslant 1$, the solution (6.2) describes a BB with an event horizon [6]. On the other hand, when $c<0$ the solution has no horizon and depending on the value of $w$ it has different asymptotic behaviour. For $0 \leqslant w<1 /(d+2)$ at $r \rightarrow \infty$ the second term in the metric function $U$ dominates over the first. Asymptotically at 
$r \rightarrow \infty$ (corresponding to $\phi \rightarrow \infty) V$ approaches to zero and the solution becomes the CL brane solution discussed in the previous section. Conversely, near $r=0$ (corresponding to $\phi=-\infty) V$ diverges, the first term dominates over the second and the solution becomes a DW. Thus the global solution (6.2) interpolates between a CL brane in the UV and a DW in the IR. Physically, this means that the UV behaviour is dominated by the kinetic energy of the scalar field, whereas the IR behaviour is dominated by the potential energy of the scalar. Obviously, for $c<0$ and $1 /(d+2)<w \leqslant 1$ the picture is reversed and we have a global solution interpolating between a CL in the IR and a DW in the UV. The solution (6.2) in the $c=0$ extremal case has been extensively investigated in ref. [70]. In the extremal case the solution becomes the DW one obtains in the $r=0$ region.

For $c>0$, solution (6.2) describes a BB and we can associate to it thermodynamical parameters. Using eq. (4.6) we can compute the total mass of the BB. In particular for the range of parameters for which the solution describes a BB (namely $c>0$ and $1 /(d+2)<w \leqslant 1)$, we find: $M=4 \pi A d w c / r_{0}$. The temperature $T$ and the entropy $S$ of the BB solution can be calculated using the well-known formulæ $T=U^{\prime}\left(r_{h}\right) / 4 \pi$ and $S=16 \pi^{2} R^{d}\left(r_{h}\right)$ :

$$
T=\frac{A[(d+2) w-1]}{4 \pi r_{0}} c^{\frac{2 w-1}{(d+2) w-1}}, \quad S=16 \pi^{2} c^{\frac{d w}{(d+2) w-1}} .
$$

Using these equations it is easy to verify that the first principle $d M=T d S$ is satisfied. Notice that in the extremal limit $c=0$ the BB becomes, as expected in view of its IR behaviour, a DW.

\section{Conformal-Lifshitz/Conformal-Lifshitz interpolating solutions}

In section 5.2 we have seen that CL-CL interpolating solutions require charged branes $(Q \neq 0)$ and a potential having the same qualitative behaviour of (6.1). Considering for simplicity the four-dimensional case, $d=2$, we take a non-vanishing electric charge and the potential

$$
V(\phi)=\frac{2 Q^{2}(1-w)}{1-3 w} e^{-4 \sqrt{\frac{w}{1-w}} \phi} .
$$

The general brane solutions are given by [6]:

$$
\begin{aligned}
U & =\frac{2 Q^{2} r_{0}^{2}}{(1-2 w)(1-3 w)}\left(\frac{r}{r_{0}}\right)^{2-4 w}\left[1-C\left(\frac{r}{r_{0}}\right)^{-1+2 w}\right], \\
R^{2} & =\left(\frac{r}{r_{0}}\right)^{2 w}, \quad \phi=\sqrt{w-w^{2}} \log \left(\frac{r}{r_{0}}\right), \quad 0 \leqslant w \leqslant 1 .
\end{aligned}
$$

Also in this case, for $C>0$ and $0 \leqslant w<1 / 2$ (with $w \neq 1 / 3$ ) the solution (7.2) describes a BB with an event horizon. For $C<0$ the brane has no horizon and we have a brane interpolating between two CL elementary solutions in the IR and UV regions. When $C<0$ and $w>1 / 2$, for $r \rightarrow \infty(\phi \rightarrow \infty)$ the potential approaches to zero and the solution gives an elementary CL solution, which coincides with the $r \rightarrow \infty$ (and $w>1 / 2$ ) regime of the 
electrically charged brane (4.17). Also near $r=0$ the solution reduces to an elementary CL brane, but with a different dynamical exponent. For $C<0$ and $0 \leqslant w<1 / 2(w \neq 1 / 3)$ we have the same limiting elementary CL branes but with the IR and UV regions exchanged.

When the solution (7.2) describes BBs $(C>0)$ we can compute the associated thermodynamical parameters:

$$
M=\frac{8 \pi B w}{r_{0}} C, \quad T=\frac{B(1-2 w)}{4 \pi r_{0}} C^{\frac{1-4 w}{1-2 w}}, \quad S=16 \pi^{2} C^{\frac{2 w}{1-2 w}},
$$

and check that the first principle $d M=T d S$ is satisfied. It is interesting to notice that the $C=0$ extremal limit of these BB solutions is described by a CL brane.

CL-CL interpolating solutions appear also in the context of non-minimally coupled Einstein-Maxwell-scalar gravity. One interesting example of CL-CL interpolating solution is derived and discussed in section 8 of ref. [70].

\section{Conclusions}

In this paper we have derived brane solutions of MCEMSG in $d+2$ dimensions in the case of a vanishing potential. We have shown that these brane solutions belong to the broad class of scale-covariant metrics, which generate hyperscaling violation in the holographically dual QFT. Moreover, these solutions can be considered as the brane counterpart of the well-known JNWW, spherically symmetric, solutions of Einstein-scalar gravity with $V=0$. We have also explicitly shown that our brane solution, together with the AdS brane and the DW solution sourced by an exponential potential, give all the possible scale-covariant, hyperscaling violating, geometries of MCEMSG with no Schrödinger isometries. Using this result we have been able to give a classification of the brane solutions of the theory in terms of symmetric (elementary) and interpolating solutions, which can be very useful for holographic applications. In particular, the interpolating solutions can find a broad field of holographic applications because the dual QFT describes the flow from different regimes (fixed points, hyperscaling violation, Lifshitz) in the UV and IR, characterized by different scaling symmetries.

In this context is important to stress the fact that some of our solutions have curvature singularities at $r=0$ (in the IR of the dual QFT). The issue of the acceptability of naked singularities in the context of holographic models and in particular in Einstein-Maxwellscalar models has been discussed in several papers [70-73]. A basic requirement for a solution with a naked singularity to be acceptable is that the scalar potential is bounded from above when evaluated in the solution [71]. This basic requirement is trivially satisfied for the brane solutions sourced by a scalar field with $V=0$, which we have discussed in section 4 . On the other hand, further requirements involving properties of the spectrum of small fluctuations near the solution must be imposed if the solution has to be considered physically acceptable $[70,72,73]$.

Alternatively, one can resolve the singularities looking for an IR completion of the theory. From the bulk point of view this completion can be realized using an IR regular geometry such as $\mathrm{AdS}_{d+2}$ [10] or $\mathrm{AdS}_{2} \times R_{d}[27]$. 


\section{A Spherically symmetric solutions in four dimensions}

This paper has been focused on brane solutions, i.e. on solutions with $\varepsilon=0$ in the field equations (2.5). However, our method for solving the field equations described in section 4 can be also used to derive solutions having $d$-dimensional sections with spherical topology, i.e. solutions with $\varepsilon=1$, sourced by a scalar field with vanishing potential. For spherically symmetric solutions in $d=2$, eq. (4.1) become

$$
Y^{\prime}+Y^{2}=-\left(\phi^{\prime}\right)^{2}, \quad\left(u \phi^{\prime}\right)^{\prime}=0, \quad u^{\prime \prime}-4(u Y)^{\prime}=-2+4 Q^{2} e^{-2 \int Y}, \quad u^{\prime \prime}=2 .
$$

The uncharged $Q=0$ solution of the previous equation gives the well-known JNWW solution $[54,55]$. The derivation of this solution using field equations in the form (A.1) has been already discussed in ref. [56]. For the $Q \neq 0$, charged case we solve eqs. (A.1) by determining first $u$ and $\phi$ then by solving the Riccati equation. Finally, we use the third equation to express the $Q$ in terms of the integration constants. We have

$$
\begin{aligned}
U & =\left(1+\frac{r_{0}}{r}\right)^{2 w-1}\left[1-\frac{Q^{2}}{r_{0}^{2}(1-2 w)^{2}}\left(1+\frac{r_{0}}{r}\right)^{2 w-1}\right]^{-2}, \\
R^{2} & =r^{2}\left(1+\frac{r_{0}}{r}\right)^{2(1-w)}\left[1-\frac{Q^{2}}{r_{0}^{2}(1-2 w)^{2}}\left(1+\frac{r_{0}}{r}\right)^{2 w-1}\right]^{2}, \\
\phi & =-\gamma \log \left(1+\frac{r_{0}}{r}\right)+\phi_{0} .
\end{aligned}
$$

with $w-w^{2}=\gamma^{2}$. For $w \neq 0,1$, these solutions can be considered as the charged generalization of the JNWW solutions and describe a spacetime with a naked singularity at $r=r_{0}\left[\left(\frac{Q}{r_{0}(1-2 w)}\right)^{\frac{2}{1-2 w}}-1\right]^{-1}$. In fact the scalar curvature of the spacetime is given by:

$$
\mathcal{R}=\frac{2 \gamma^{2} r_{0}^{2}}{r^{4}}\left(1+\frac{r_{0}}{r}\right)^{2 w-3}\left[1-\frac{Q^{2}}{r_{0}^{2}(1-2 w)^{2}}\left(1+\frac{r_{0}}{r}\right)^{2 w-1}\right]^{-2} .
$$

For $w=0,1$ the solution gives the usual $\mathrm{RN}$ black hole solution with a constant scalar field $\phi$. In fact in these cases the previous solution can be put in the usual RN form by rescaling and translating the radial coordinate $r$.

\section{Acknowledgments}

EF acknowledges financial support provided under the European Union's H2020 ERC Consolidator Grant "Matter and strong-field gravity: New frontiers in Einstein's theory" grant agreement no. MaGRaTh-646597.

Open Access. This article is distributed under the terms of the Creative Commons Attribution License (CC-BY 4.0), which permits any use, distribution and reproduction in any medium, provided the original author(s) and source are credited. 


\section{References}

[1] C. Charmousis, B. Gouteraux and J. Soda, Einstein-Maxwell-dilaton theories with a Liouville potential, Phys. Rev. D 80 (2009) 024028 [arXiv:0905.3337] [INSPIRE].

[2] S.S. Gubser and F.D. Rocha, Peculiar properties of a charged dilatonic black hole in AdS $S_{5}$, Phys. Rev. D 81 (2010) 046001 [arXiv:0911.2898] [InSPIRE].

[3] K. Goldstein, S. Kachru, S. Prakash and S.P. Trivedi, Holography of charged dilaton black holes, JHEP 08 (2010) 078 [arXiv:0911.3586] [INSPIRE].

[4] M. Cadoni, G. D'Appollonio and P. Pani, Phase transitions between Reissner-Nordstrom and dilatonic black holes in 4D AdS spacetime, JHEP 03 (2010) 100 [arXiv:0912.3520] [INSPIRE].

[5] G. Bertoldi, B.A. Burrington, A.W. Peet and I.G. Zadeh, Lifshitz-like black brane thermodynamics in higher dimensions, Phys. Rev. D 83 (2011) 126006 [arXiv:1101.1980] [INSPIRE].

[6] M. Cadoni, S. Mignemi and M. Serra, Exact solutions with AdS asymptotics of Einstein and Einstein-Maxwell gravity minimally coupled to a scalar field, Phys. Rev. D 84 (2011) 084046 [arXiv:1107.5979] [INSPIRE].

[7] B. Gouteraux, J. Smolic, M. Smolic, K. Skenderis and M. Taylor, Holography for Einstein-Maxwell-dilaton theories from generalized dimensional reduction, JHEP 01 (2012) 089 [arXiv:1110.2320] [INSPIRE].

[8] M. Cadoni, S. Mignemi and M. Serra, Black brane solutions and their solitonic extremal limit in Einstein-scalar gravity, Phys. Rev. D 85 (2012) 086001 [arXiv:1111.6581] [InSPIRE].

[9] M. Cadoni and S. Mignemi, Phase transition and hyperscaling violation for scalar black branes, JHEP 06 (2012) 056 [arXiv: 1205.0412] [INSPIRE].

[10] M. Cadoni and M. Serra, Hyperscaling violation for scalar black branes in arbitrary dimensions, JHEP 11 (2012) 136 [arXiv: 1209.4484] [INSPIRE].

[11] B. Gouteraux and E. Kiritsis, Quantum critical lines in holographic phases with (un)broken symmetry, JHEP 04 (2013) 053 [arXiv: 1212.2625] [INSPIRE].

[12] M. Cadoni, P. Pani and M. Serra, Infrared behavior of scalar condensates in effective holographic theories, JHEP 06 (2013) 029 [arXiv: 1304.3279] [INSPIRE].

[13] J.M. Maldacena, The large-N limit of superconformal field theories and supergravity, Int. J. Theor. Phys. 38 (1999) 1113 [hep-th/9711200] [InSPIRE].

[14] G.T. Horowitz and J. Polchinski, Gauge/gravity duality, in Approaches to quantum gravity, D. Oriti ed. Cambridge University Press, Cambridge U.K. (2009), gr-qc/0602037 [INSPIRE].

[15] S.A. Hartnoll, Lectures on holographic methods for condensed matter physics, Class. Quant. Grav. 26 (2009) 224002 [arXiv:0903.3246] [INSPIRE].

[16] S.A. Hartnoll, C.P. Herzog and G.T. Horowitz, Building a holographic superconductor, Phys. Rev. Lett. 101 (2008) 031601 [arXiv:0803.3295] [INSPIRE].

[17] G.T. Horowitz and M.M. Roberts, Holographic superconductors with various condensates, Phys. Rev. D 78 (2008) 126008 [arXiv:0810.1077] [INSPIRE].

[18] C.P. Herzog, Lectures on holographic superfluidity and superconductivity, J. Phys. A 42 (2009) 343001 [arXiv:0904.1975] [InSPIRE]. 
[19] G.T. Horowitz, Introduction to holographic superconductors, Lect. Notes Phys. 828 (2011) 313 [arXiv:1002.1722].

[20] M. Cadoni and P. Pani, Holography of charged dilatonic black branes at finite temperature, JHEP 04 (2011) 049 [arXiv: 1102.3820] [INSPIRE].

[21] S.S. Gubser and F.D. Rocha, The gravity dual to a quantum critical point with spontaneous symmetry breaking, Phys. Rev. Lett. 102 (2009) 061601 [arXiv:0807.1737] [INSPIRE].

[22] B. Gouteraux and E. Kiritsis, Generalized holographic quantum criticality at finite density, JHEP 12 (2011) 036 [arXiv:1107.2116] [INSPIRE].

[23] X. Dong, S. Harrison, S. Kachru, G. Torroba and H. Wang, Aspects of holography for theories with hyperscaling violation, JHEP 06 (2012) 041 [arXiv:1201.1905] [INSPIRE].

[24] K. Narayan, On Lifshitz scaling and hyperscaling violation in string theory, Phys. Rev. D 85 (2012) 106006 [arXiv:1202.5935] [INSPIRE].

[25] E. Perlmutter, Hyperscaling violation from supergravity, JHEP 06 (2012) 165 [arXiv: 1205.0242] [INSPIRE].

[26] M. Ammon, M. Kaminski and A. Karch, Hyperscaling-violation on probe D-branes, JHEP 11 (2012) 028 [arXiv:1207.1726] [INSPIRE].

[27] J. Bhattacharya, S. Cremonini and A. Sinkovics, On the IR completion of geometries with hyperscaling violation, JHEP 02 (2013) 147 [arXiv: 1208.1752] [INSPIRE].

[28] M. Alishahiha and H. Yavartanoo, On holography with hyperscaling violation, JHEP 11 (2012) 034 [arXiv:1208.6197] [INSPIRE].

[29] P. Dey and S. Roy, Lifshitz metric with hyperscaling violation from NS5-Dp states in string theory, Phys. Lett. B 720 (2013) 419 [arXiv:1209.1049] [InSPIRE].

[30] J. Sadeghi, B. Pourhasan and F. Pourasadollah, Thermodynamics of Schrödinger black holes with hyperscaling violation, Phys. Lett. B 720 (2013) 244 [arXiv:1209.1874] [INSPIRE].

[31] M. Alishahiha, E. O Colgain and H. Yavartanoo, Charged black branes with hyperscaling violating factor, JHEP 11 (2012) 137 [arXiv: 1209.3946] [INSPIRE].

[32] B.S. Kim, Hyperscaling violation : a unified frame for effective holographic theories, JHEP 11 (2012) 061 [arXiv:1210.0540] [INSPIRE].

[33] M. Edalati, J.F. Pedraza and W. Tangarife Garcia, Quantum fluctuations in holographic theories with hyperscaling violation, Phys. Rev. D 87 (2013) 046001 [arXiv:1210.6993] [INSPIRE].

[34] J. Gath, J. Hartong, R. Monteiro and N.A. Obers, Holographic models for theories with hyperscaling violation, JHEP 04 (2013) 159 [arXiv: 1212.3263] [INSPIRE].

[35] S. Cremonini and A. Sinkovics, Spatially modulated instabilities of geometries with hyperscaling violation, JHEP 01 (2014) 099 [arXiv: 1212.4172] [INSPIRE].

[36] M. Hassaïne, New black holes of vacuum Einstein equations with hyperscaling violation and nil geometry horizons, Phys. Rev. D 91 (2015) 084054 [arXiv:1503.01716] [INSPIRE].

[37] M. Bravo-Gaete, S. Gomez and M. Hassaine, Towards the Cardy formula for hyperscaling violation black holes, Phys. Rev. D 91 (2015) 124038 [arXiv:1505.00702] [INSPIRE].

[38] G. Kofinas, Hyperscaling violating black holes in scalar-torsion theories, Phys. Rev. D 92 (2015) 084022 [arXiv:1507.07434] [InSPIRE]. 
[39] A. Salvio, Holographic superfluids and superconductors in dilaton-gravity, JHEP 09 (2012) 134 [arXiv:1207.3800] [INSPIRE].

[40] D. Roychowdhury, Hydrodynamics from scalar black branes, JHEP 04 (2015) 162 [arXiv: 1502.04345] [INSPIRE].

[41] N. Ogawa, T. Takayanagi and T. Ugajin, Holographic Fermi surfaces and entanglement entropy, JHEP 01 (2012) 125 [arXiv:1111.1023] [INSPIRE].

[42] K. Narayan, T. Takayanagi and S.P. Trivedi, AdS plane waves and entanglement entropy, JHEP 04 (2013) 051 [arXiv: 1212.4328] [INSPIRE].

[43] K. Narayan, Non-conformal brane plane waves and entanglement entropy, Phys. Lett. B 726 (2013) 370 [arXiv:1304.6697] [INSPIRE].

[44] S. Cremonini and X. Dong, Constraints on renormalization group flows from holographic entanglement entropy, Phys. Rev. D 89 (2014) 065041 [arXiv:1311.3307] [INSPIRE].

[45] K. Skenderis and P.K. Townsend, Hidden supersymmetry of domain walls and cosmologies, Phys. Rev. Lett. 96 (2006) 191301 [hep-th/0602260] [INSPIRE].

[46] E. Shaghoulian, FRW cosmologies and hyperscaling-violating geometries: higher curvature corrections, ultrametricity, Q-space/QFT duality and a little string theory, JHEP 03 (2014) 011 [arXiv: 1308.1095] [INSPIRE].

[47] M. Cadoni and M. Ciulu, Dark energy from holographic theories with hyperscaling violation, JHEP 05 (2014) 089 [arXiv: 1311.4098] [INSPIRE].

[48] S. Mignemi and N. Pintus, An exactly solvable inflationary model, Gen. Rel. Grav. 47 (2015) 51 [arXiv: 1404.4720] [INSPIRE].

[49] M. Cadoni, E. Franzin and S. Mignemi, Inflation as de Sitter instability, arXiv:1510.04030 [INSPIRE].

[50] M. Cadoni, Scalar black branes with non-AdS asymptotics, in the proceedings of the $13^{\text {th }}$ Marcel Grossmann Meeting (MG13), July 1-7, Stockholm, Sweden (2012), R. Ruffini et al. eds., World Scientific (2013), arXiv: 1305.7379 [INSPIRE].

[51] W. Israel, Event horizons in static vacuum space-times, Phys. Rev. 164 (1967) 1776 [INSPIRE].

[52] J.D. Bekenstein, Novel 'no scalar hair' theorem for black holes, Phys. Rev. D 51 (1995) 6608 [INSPIRE].

[53] T. Hertog, Towards a novel no-hair theorem for black holes, Phys. Rev. D 74 (2006) 084008 [gr-qc/0608075] [INSPIRE].

[54] A.I. Janis, E.T. Newman and J. Winicour, Reality of the Schwarzschild singularity, Phys. Rev. Lett. 20 (1968) 878 [INSPIRE].

[55] M. Wyman, Static spherically symmetric scalar fields in general relativity, Phys. Rev. D 24 (1981) 839 [INSPIRE].

[56] M. Cadoni and E. Franzin, Asymptotically flat black holes sourced by a massless scalar field, Phys. Rev. D 91 (2015) 104011 [arXiv: 1503.04734] [INSPIRE].

[57] M. Cadoni and E. Franzin, Black holes sourced by a massless scalar, arXiv:1510.02076 [INSPIRE]. 
[58] D.T. Son, Toward an AdS/cold atoms correspondence: a geometric realization of the Schrödinger symmetry, Phys. Rev. D 78 (2008) 046003 [arXiv: 0804.3972] [INSPIRE].

[59] K. Balasubramanian and J. McGreevy, Gravity duals for non-relativistic CFTs, Phys. Rev. Lett. 101 (2008) 061601 [arXiv:0804.4053] [INSPIRE].

[60] C. Duval, M. Hassaine and P.A. Horvathy, The geometryc of Schrödinger symmetry in gravity background/non-relativistic CFT, Annals Phys. 324 (2009) 1158 [arXiv:0809.3128] [INSPIRE].

[61] J. Hartong, E. Kiritsis and N.A. Obers, Lifshitz space-times for Schrödinger holography, Phys. Lett. B 746 (2015) 318 [arXiv:1409.1519] [INSPIRE].

[62] J. Hartong, E. Kiritsis and N.A. Obers, Schrödinger Invariance from Lifshitz Isometries in Holography and Field Theory, Phys. Rev. D 92 (2015) 066003 [arXiv: 1409.1522] [INSPIRE].

[63] T. Andrade, C. Keeler, A. Peach and S.F. Ross, Schrödinger holography with $z=2$, Class. Quant. Grav. 32 (2015) 085006 [arXiv:1412.0031] [INSPIRE].

[64] H.J. Boonstra, K. Skenderis and P.K. Townsend, The domain wall/QFT correspondence, JHEP 01 (1999) 003 [hep-th/9807137] [INSPIRE].

[65] I. Kanitscheider and K. Skenderis, Universal hydrodynamics of non-conformal branes, JHEP 04 (2009) 062 [arXiv: 0901.1487] [INSPIRE].

[66] C. Martinez, R. Troncoso and J. Zanelli, Exact black hole solution with a minimally coupled scalar field, Phys. Rev. D 70 (2004) 084035 [hep-th/0406111] [INSPIRE].

[67] C. Martinez and R. Troncoso, Electrically charged black hole with scalar hair, Phys. Rev. D 74 (2006) 064007 [hep-th/0606130] [INSPIRE].

[68] J. de Boer, The Holographic renormalization group, in the proceedings of the Workshop on the quantum structure of space-time and the geometrical nature of fundamental interactions, October 4-10, Berlin, Germany (2000), E.A. Bergshoeff et al. eds., Wiley (2001), hep-th/0101026 [INSPIRE].

[69] S.S. Gubser, S.S. Pufu and F.D. Rocha, Quantum critical superconductors in string theory and M-theory, Phys. Lett. B 683 (2010) 201 [arXiv:0908.0011] [INSPIRE].

[70] C. Charmousis, B. Gouteraux, B.S. Kim, E. Kiritsis and R. Meyer, Effective holographic theories for low-temperature condensed matter systems, JHEP 11 (2010) 151 [arXiv: 1005.4690] [INSPIRE].

[71] S.S. Gubser, Curvature singularities: the good, the bad and the naked, Adv. Theor. Math. Phys. 4 (2000) 679 [hep-th/0002160] [INSPIRE].

[72] U. Gürsoy, E. Kiritsis and F. Nitti, Exploring improved holographic theories for QCD: part II, JHEP 02 (2008) 019 [arXiv: 0707.1349] [INSPIRE].

[73] U. Gürsoy, E. Kiritsis, L. Mazzanti and F. Nitti, holography and thermodynamics of 5D dilaton-gravity, JHEP 05 (2009) 033 [arXiv:0812.0792] [INSPIRE]. 